\title{
Asymptotic Behavior of Vector Recurrences with Applications
}

\author{
By Alan Feldstein and J. F. Traub*
}

\begin{abstract}
The behavior of the vector recurrence $y_{n+1}=M y_{n}+w_{n+1}$ is studied under very weak assumptions. Let $\lambda(M)$ denote the spectral radius of $M$ and let $\lambda(M) \geqslant 1$. Then if the $w_{n}$ are bounded in norm and a certain subspace hypothesis holds, the root order of the $y_{n}$ is shown to be $\lambda(M)$. If one additional hypothesis on the dimension of the principal Jordan blocks of $M$ holds, then the quotient order of the $y_{n}$ is also $\lambda(M)$. The behavior of the homogeneous recurrence is studied for all values of $\lambda(M)$.

These results are applied to the analysis of

(1) Nonlinear iteration with application to iteration with memory and to parallel iteration algorithms.

(2) Order and efficiency of composite iteration.
\end{abstract}

1. Introduction. We study the behavior of the vector recurrence

$$
\mathrm{y}_{n+1}=M \mathrm{y}_{n}+\mathrm{w}_{n+1}
$$

under very weak assumptions. We apply our results to the analysis of iterations for nonlinear equations and to the composition of such iterations. In particular our results can be used to study one-point iterations with memory and iterations for solving nonlinear equations on parallel computers. An extended discussion of applications, including use of the power method to calculate the spectral radius, may be found in Feldstein and Traub [74].

Let $\|\cdot\|$ denote any convenient vector norm or the induced matrix norm. When the following limits exist, define the root order by

and the quotient order by

$$
R\left(\mathrm{y}_{n}\right)=\lim _{n \rightarrow \infty}\left\|\mathrm{y}_{n}\right\|^{1 / n}
$$

$$
Q\left(\mathrm{y}_{n}\right)=\lim _{n \rightarrow \infty} \frac{\left\|\mathrm{y}_{n+1}\right\|}{\left\|\mathrm{y}_{n}\right\|} .
$$

Clearly, if the quotient order exists, then so does the root order (though not conversely), and they are equal.

Received February 21, 1975; revised August 26, 1975 and May 27, 1976. $65 \mathrm{H} 05,68 \mathrm{~A} 20$.

AMS (MOS) subject classifications (1970). Primary 39A10, 40A05, 65 F15, 15A18,

* Part of the first author's work was done while in residence at the Naval Research Laboratory, Washington, D. C. and at ICASE, NASA-Langley Research Center under NASA Grant NGR 47-102-001. Part of the second author's work was performed under the auspices of the U. S. Atomic Energy Commission while consulting at Lawrence Livermore Laboratory. The second author was supported in part by the National Science Foundation under Grant GJ 32111 and the Office of Naval Research under Contract N0014-67-A-0314-0010, NR 044-422. 
Let $U$ be a nonsingular matrix such that $M=U^{-1} J U$, where $J$ is the direct sum of $K$ Jordan block matrices, $J=J_{1} \oplus J_{2} \oplus \cdots \oplus J_{K}$. Let $\lambda_{k}$ be the eigenvalue corresponding to $J_{k}$ and let the dimension of $J_{k}$ be $D_{k}$. Let the $K$ Jordan blocks of $J$ be arranged so that

$$
\left\{\begin{array}{l}
\left|\lambda_{1}\right| \geqslant\left|\lambda_{2}\right| \geqslant \cdots \geqslant\left|\lambda_{K}\right|, \text { and } \\
\left|\lambda_{1}\right|=\left|\lambda_{2}\right|=\cdots=\left|\lambda_{L}\right| \text { implies } D_{1} \geqslant D_{2} \geqslant \cdots \geqslant D_{L} .
\end{array}\right.
$$

$J_{1}, J_{2}, \ldots, J_{L}$ are called the principal Jordan blocks of $M$. Denote $D=D_{1}$, and $\lambda=\lambda_{1}$. Thus $|\lambda|$ is the spectral radius of $M$, which we shall sometimes write as $\lambda(M)$.

In order to draw the conclusions which follow we must assume that the initial vector $\mathbf{y}_{0}$ does not lie in a certain subspace. Since the statement of this hypothesis is given in Eq. (16) and involves certain quantities not defined until Section 5, we find it convenient to label this as the "subspace hypothesis". We now state our main result; the proof is given in Section 5 .

THEOREM 1. Assume $\lambda(M) \geqslant 1$ and

1. $\left\|\mathrm{w}_{n}\right\| \leqslant w<\infty$ for all $n$,

2. "Subspace hypothesis".

Then

(i) $R\left(\mathrm{y}_{n}\right)=\lambda(M)$.

If , in addition,

3. $D_{k}<D$ for $2 \leqslant k \leqslant L$, when $\left|\lambda_{k}\right|=|\lambda|$ with $\lambda_{k} \neq \lambda$, then

(ii) $Q\left(\mathrm{y}_{n}\right)=\lambda(M)$.

Herzberger [74] has independently analyzed the order of (1). However, his assumptions are far more restrictive than ours. Of course there are important applications of (1) where Herzberger's conditions hold. In our terminology Herzberger's main result may be stated as

Theorem 1'. Assume

1. $\lim _{n \rightarrow \infty} \mathbf{w}_{n}=\mathbf{w}<\infty$,

2. $\lambda(M)>1$,

3. $M$ is a nonnegative matrix,

4. $M$ is primitive (see Varga [62]).

Then

$$
R\left(\mathrm{y}_{n}\right)=Q\left(\mathrm{y}_{n}\right)=\lambda(M)
$$

Because of his strong conditions, Herzberger does not need to distinguish between the existence of root and quotient order. Herzberger does not include a subspace hypothesis although we believe one to be necessary.

The example $\mathrm{y}_{n+1}=\lambda \mathrm{y}_{n}+\mathrm{w}, \lambda<1, w \neq 0$, shows that Theorem 1 need not hold if $\lambda<1$. However the conclusions of Theorem 1 hold for all values of $\lambda$ if we restrict ourselves to homogeneous recurrences, $w_{n}=0$ for all $n$. In this case (1) becomes $\mathbf{y}_{n+1}=M y_{n+1}$, the $y_{n}$ are the iterates of the power method and we have

THEOREM 2. Assume

1. $\mathbf{w}_{n}=\mathbf{0}$ for all $n$. 
If $\lambda(M)=0$,

(i) $R\left(\mathrm{y}_{n}\right)=\lambda(M)$.

If $\lambda(M)>0$ and if

(2) "Homogeneous subspace hypothesis", then

(ii) $R\left(\mathrm{y}_{n}\right)=\lambda(M)$.

If, in addition,

3. $D_{k}<D$ for $2 \leqslant k \leqslant L$, when $\left|\lambda_{k}\right|=\lambda$ with $\lambda_{k}=\lambda$, then

(iii) $Q\left(\mathrm{y}_{n}\right)=\lambda(M)$.

Observe that if $\lambda(M)=0$, then no subspace condition is required. If $\lambda(M)>0$, then the homogeneous subspace hypothesis is the classical condition that the initial vector $\mathbf{y}_{\mathbf{0}}$ may not be an eigenvector corresponding to a subdominant eigenvalue. In the notation of this paper the homogeneous subspace hypothesis is (16) with $\mathbf{f}=\mathbf{0}$ and $\delta=1$ for all $\lambda$ (even $|\lambda|=1)$.

We summarize the rest of this paper. Applications are considered in the next two sections while the proof of the main theorem is deferred until the last two sections. Readers interested primarily in the proof should turn first to Section 4 . Section 2 discusses the matrix representation of nonlinear iteration and utilizes the representation in the analysis of parallel algorithms. New results on the order and efficiency of composite iteration are analyzed in Section 3. The main result is proved in Section 4. Proofs of estimates needed in the proof of the main theorem may be of independent interest and appear in Section 5.

\section{Matrix Representation of Nonlinear Iteration: Applications to Iteration}

With Memory and to Parallel Algorithms. Let a sequence of vectors $\left\{\mathbf{x}_{n}\right\}$ be generated by the vector-valued function $\varphi: R^{N} \rightarrow R^{N}$ and

$$
\mathbf{x}_{n+1}=\varphi\left(\mathbf{x}_{n}\right) .
$$

Assume that at least one component of $\mathbf{x}_{n}$ converges to at least one component of the constant vector $\alpha$. Let the components of $x_{n}, y_{n}$, and $\alpha$ be labelled $x_{n, j}, y_{n, j}$, and $\alpha_{j}$. Let

$$
y_{n, j}=\log \left|x_{n, j}-\alpha_{j}\right|
$$

Then $\left\|y_{n}\right\| \rightarrow \infty$ as $n \rightarrow \infty$. For many important problems, the vectors $\mathbf{y}_{n}$ satisfy (1). Examples are given below. We then call (1) the logarithmic error equation (or simply the error equation) for the sequence $\left\{\mathbf{x}_{n}\right\}$ and call $M$ the matrix representation of the iteration function $\varphi$.

If (1) is the error equation of (2), we define the root order of $\varphi$ as

$$
R(\varphi)=R\left(\mathrm{y}_{n}\right)
$$

and the quotient order of $\varphi$ as

$$
Q(\varphi)=Q\left(\mathbf{y}_{n}\right)
$$

The important idea of matrix representation of nonlinear iteration is due to 
Rice [71]. His matrix representation seems unnecessarily complicated. Rice's analysis does not distinguish between root and quotient order.

The following example shows applications of this formulation.

Example 1. Assume that $\alpha$ is a zero of a scalar function $g$. One-point iterations with memory are of the form (Traub [64], Feldstein and Firestone [67] and [69], Hindmarsh [72])

$$
z_{n+1}=\varphi\left(z_{n}, z_{n-1}, \ldots, z_{n-N+1}\right)
$$

with errors satisfying

$$
z_{n+1}-\alpha=c_{n+1}\left(z_{n}-\alpha\right)^{b_{1}} \cdots\left(z_{n-N+1}-\alpha\right)^{b_{N}},
$$

where the $b_{i}$ are nonnegative integers.

Equation (3) may be written as

$$
\left\{\begin{array}{l}
x_{n+1,1}=\varphi\left(x_{n, 1}, \ldots, x_{n, N}\right), \\
x_{n+1, j}=x_{n, j-1} \quad \text { for } j=2,3, \ldots, N
\end{array}\right.
$$

Let all components of $\alpha$ be $\alpha$. One easily shows that the error $y_{n+1}$ satisfies

$$
\mathrm{y}_{n+1}=M \mathrm{y}_{n}+\mathrm{w}_{n+1} \text { where }
$$

$$
M=\left(\begin{array}{ccccc}
b_{1} & b_{2} & \cdots & b_{N-1} & b_{N} \\
1 & 0 & & & \\
0 & 1 & . & & \\
\cdot & & . & & \\
\cdot & & & & \\
\cdot & & & & \\
0 & 0 & & 1 & 0
\end{array}\right), \quad \mathbf{w}_{n+1}=\left(\begin{array}{c}
\log \left|c_{n+1}\right| \\
0 \\
0 \\
\cdot \\
\cdot \\
\cdot \\
0
\end{array}\right)
$$

Observe that $M$ is the companion matrix for the indicial polynomial of the linear recurrence.

Conclusions (i) and (ii) of Theorem 1 hold for all Hermite interpolatory iteration functions. The quotient order was first established by Traub [64] for the equal information case $b_{1}=b_{2}=\cdots=b_{N}$ and by Feldstein and Firestone [67] for arbitrary nonnegative integers $b_{1}, \ldots, b_{N}$ using recurrence equation techniques.

A number of authors (Feldstein and Firestone [67], Shedler [67], Miranker [69] and Rice [71]) have studied the use of parallel computers to calculate a zero of a scalar function. Most of the proposed iterations have the form

$$
x_{n+1, i}=\varphi_{i}\left(x_{n, 1}, \ldots, x_{n, N}\right) \text { for } i=1, \ldots, N,
$$

where $N$ is the number of processors. Assume the errors satisfy

$$
x_{n+1, i}-\alpha=c_{n, i} \prod_{j=1}^{N}\left(x_{n, j}-\alpha\right)^{m_{i, j}}
$$


Then the error equation (1) holds with

$$
\mathbf{w}_{n}=\left(\log \left|c_{n, 1}\right|, \ldots, \log \left|c_{n, N}\right|\right)^{T}
$$

and with the elements of $M$ given by $\left(m_{i, j}\right)$. See Examples 3 and 5 of Feldstein and Traub [74] for more details.

3. Composition and Efficiency. We turn to the order of a composite iteration. Suppose that

$$
\mathbf{x}_{n+1}^{(1)}=\varphi_{1}\left(\mathbf{x}_{n}^{(1)}\right), \quad \mathbf{x}_{n+1}^{(2)}=\varphi_{2}\left(\mathbf{x}_{n}^{(2)}\right) .
$$

Then the composite iteration is $\psi=\varphi_{2} \circ \varphi_{1}$, where $\psi: R^{N} \rightarrow R^{N}$, and the composite iteration sequence $\left\{\mathbf{x}_{n}\right\}$ is defined by

$$
\mathbf{x}_{n+1}=\psi\left(x_{n}\right) \equiv \varphi_{2}\left(\varphi_{1}\left(\mathbf{x}_{n}\right)\right)
$$

Let $\varphi_{1}$ and $\varphi_{2}$ have characteristic matrices $M_{1}$ and $M_{2}$ with logarithmic error equations

$$
\mathrm{y}_{n+1}^{(1)}=M_{1} \mathrm{y}_{n}^{(1)}+\mathrm{w}_{n+1}^{(1)}, \quad \mathrm{y}_{n+1}^{(2)}=M_{2} \mathrm{y}_{n}^{(2)}+\mathrm{w}_{n+1}^{(2)} .
$$

Let $M=M_{2} M_{1}$ and $\mathrm{w}_{n+1}=M_{2} \mathrm{w}_{n+1}^{(1)}+\mathrm{w}_{n+1}^{(2)}$. Then $\psi$ has the error equation $\mathbf{y}_{n+1}=M y_{n}+w_{n+1}$. See Example 6 of Feldstein and Traub [74] for specific representations of composite algorithms.

In the following theorem the use of $D, D_{k}, \lambda, \lambda_{k}$ and the subspace hypotheses all refer to the matrix $M=M_{2} M_{1}$. We state the main Composition Theorem. Its proof is an immediate consequence of Theorem 1.

Theorem 3. Assume

1. $\left\|\mathrm{w}_{n+1}\right\| \leqslant w<\infty$ for all $n$,

2. "Subspace Hypothesis",

3. $\lambda\left(M_{2} M_{1}\right) \geqslant 1$.

Then

$$
R\left(\varphi_{2} \circ \varphi_{1}\right)=\lambda\left(M_{2} M_{1}\right)
$$

If, in addition,

4. $D_{k}<D$ for $2 \leqslant k \leqslant L$, when $\left|\lambda_{k}\right|=|\lambda|$ with $\lambda_{k} \neq \lambda$, then

$$
Q\left(\varphi_{2} \circ \varphi_{1}\right)=\lambda\left(M_{2} M_{1}\right)
$$

Observe that a composite algorithm may have an order of convergence either less than, equal to, or greater than the product of the orders of the individual algorithms. See Example 7 of Feldstein and Traub [74] for details. It is easy to see that

COROLlARY 1. Order is invariant under commuting of two compositions.

Observe that Corollary 1 is false for three compositions, because $\lambda\left(M_{1} M_{2} M_{3}\right)$ need not equal $\lambda\left(M_{3} M_{2} M_{1}\right)$.

From Corollary 1, order multiplies under self-composition. Another important case for which order multiplies under composition is when $\varphi$ is a scalar one-point iteration (Traub [64, Chapter 2]). In general, order does not multiply under 
composition even in the case of scalar $\varphi$. This was first shown by Hindmarsh [72] who used difference equation techniques in his analysis, and by Rice [71] using matrix representation techniques.

To compare iterations it is useful to have the concept of an efficiency measure. See Kung and Traub [74] and Traub and Woźniakowski [76]. Let $c(\varphi)>0$ be some "cost" associated with computing $\mathbf{x}_{n+1}$ from $\mathbf{x}_{n}$. The efficiency index of $\varphi$ is defined, for $p>1$, by

$$
e(\varphi)=(\log p(\varphi)) / c(\varphi)
$$

We wish to compare the efficiency of the composite iteration $\varphi_{2} \circ \varphi_{1}$ with the efficiencies of $\varphi_{1}$ and $\varphi_{2}$. In the following discussion we do not distinguish between root and quotient order; both are represented by $p(\varphi)$. We denote $p_{i}=p\left(\varphi_{i}\right), p_{i, j}=$ $p\left(\varphi_{i} \circ \varphi_{j}\right), c_{i, j}=c\left(\varphi_{i} \circ \varphi_{j}\right), e_{i, j}=e\left(\varphi_{i} \circ \varphi_{j}\right)$. We assume that $c_{1,2}=c_{2,1}$. Then by Corollary $1, e_{1,2}=e_{2,1}$. It is reasonable to assume that $c_{2,1} \leqslant c_{1}+c_{2}$. In the following theorem assume that $p_{1}=\lambda\left(M_{1}\right), p_{2}=\lambda\left(M_{2}\right)$, and $p_{2,1}=\lambda\left(M_{2} M_{1}\right)$. The hypotheses of Theorems 1 and 3 are sufficient to guarantee this. We omit the proof which is straightforward.

THEOREM 4. 1. Let $c_{2,1}=c_{1}+c_{2}$.

(a) If $\lambda\left(M_{2} M_{1}\right)>\lambda\left(M_{2}\right) \lambda\left(M_{1}\right)$, then $\min \left(e_{1}, e_{2}\right)<e_{2,1}$.

(b) If $\lambda\left(M_{2} M_{1}\right)=\lambda\left(M_{2}\right) \lambda\left(M_{1}\right)$, then $\min \left(e_{1}, e_{2}\right) \leqslant e_{2,1} \leqslant \max \left(e_{1}, e_{2}\right)$.

(c) If $\lambda\left(M_{2} M_{1}\right)<\lambda\left(M_{2}\right) \lambda\left(M_{1}\right)$, then $e_{2,1}<\max \left(e_{1}, e_{2}\right)$.

2. On the other hand, let $c_{2,1}<c_{1}+c_{2}$.

If $\lambda\left(M_{2} M_{1}\right) \geqslant \lambda\left(M_{2}\right) \lambda\left(M_{1}\right)$, then $\min \left(e_{1}, e_{2}\right)<e_{2,1}$.

It is possible that the efficiency of a composite iteration may be greater than the maximum efficiencies of the component iterations, i.e.,

$$
e_{2,1}>\max \left(e_{1}, e_{2}\right) \text {. }
$$

In view of Theorem 4 this can only happen if either of the following conditions holds:

$$
c_{2,1}<c_{1}+c_{2}
$$

or

$$
\lambda\left(M_{2} M_{1}\right)>\lambda\left(M_{2}\right) \cdot \lambda\left(M_{1}\right) .
$$

Example 9 of Feldstein and Traub [74] shows a situation where (7a, b, c) all hold.

We can easily calculate sufficient conditions for (7a) to hold. For example, assume that $c_{2,1}=c_{1}+c_{2}$ and that $(7 \mathrm{c})$ holds. Let

$$
\eta=\log \lambda\left(M_{2} M_{1}\right)-\log \lambda\left(M_{2}\right)-\log \lambda\left(M_{1}\right)>0 .
$$

Then $e_{2,1}>\max \left(e_{1}, e_{2}\right)$ if

$$
\eta>c_{1}\left(e_{2}-e_{1}\right) \text { for } e_{2}>e_{1}, \text { or } \eta>c_{2}\left(e_{1}-e_{2}\right) \text { for } e_{2}<e_{1} \text {. }
$$

In general we are interested in iterations such that $\eta$ and $\mu=c\left(\varphi_{1}\right)+c\left(\varphi_{2}\right)-$ $c\left(\varphi_{2} \circ \varphi_{1}\right)$ are positive and as large as possible. How to do this is an open question. 
4. Proof of The Main Theorem. The proof of Theorem 1 uses certain estimates (Lemmas 1 and 2, below) on the growth as $n \rightarrow \infty$ of each block $J_{k}^{n}$ for $1 \leqslant k \leqslant K$. Since the proofs of these lemmas are rather long and since these lemmas may be of independent interest, we defer the proofs until Section 5 and confine ourselves here to a statement of the results needed for the proof of Theorem 1. We shall start from Eq. (1), written here for notational convenience with primes as

$$
\mathrm{y}_{n+1}^{\prime}=M \mathrm{y}_{n}^{\prime}+\mathrm{w}_{n+1}^{\prime} \text {. }
$$

Write $M$ in the Jordan form $M=U^{-1} J U$. Let

$$
\mathbf{y}_{n}=U \mathbf{y}_{n}^{\prime} \quad \text { and } \quad \mathbf{w}_{n}=U \mathbf{w}_{n}^{\prime} .
$$

Then

$$
\mathbf{y}_{n+1}=J \mathbf{y}_{n}+\mathbf{w}_{n+1} .
$$

Let $C(a, b)$ denote a binomial coefficient. Let $\mathbf{w}_{n, k}$ be that portion of the vector $\mathbf{w}_{n}$ which is associated with the Jordan block $J_{k}$. Let

$$
\delta= \begin{cases}0 & \text { if }|\lambda|=1 \\ 1 & \text { otherwise }\end{cases}
$$

$$
Q_{n, k}=J_{k}^{n} /\left(\lambda^{n} C(n, D-\delta)\right)
$$

$$
\mathrm{s}_{n, k}=\sum_{i=0}^{n} J_{k}^{i} \mathrm{w}_{n-i, k} .
$$

When the limit exists (see Eqs. (28) and (30)), define

$$
\mathbf{f}_{k}(z)=\lim _{n \rightarrow \infty} \sum_{i=0}^{n-D} z^{i} \frac{C(n-i, D-1)}{C(n, D-\delta)} \mathrm{w}_{i, k} .
$$

If $\left\|\mathrm{w}_{n}\right\|<w<\infty$ for all $n$, then we will show (30) that this limit exists and indeed that $\mathbf{f}_{k}(z)$ is analytic for $|z| \leqslant\left|\lambda^{-1}\right|$.

For $1 \leqslant k \leqslant K$ let $W_{k}$ be the $D_{k} \times D_{k}$ matrix having ones on the superdiagonal and zeros elsewhere. Then $w_{k}^{j}=0$ for $j \geqslant D_{k}$ and $W_{k}^{D_{k}-1}$ is a matrix with a one in the upper right-hand corner and zeros elsewhere. If $D_{k}=1$, define $W_{k}=0, w_{k}^{D_{k}-1}$ $=1$. Usually the subscript of $W_{k}$ will be clear from context, and we will simply write $W$. The symbol $O\left(n^{-1}\right)$ will denote a scalar, a vector, or a matrix (according to context) each of whose entries is bounded in absolute value by $n^{-1}$ times some nonnegative constant.

Lemma 1. Let $\lambda(M)>0$. The following hold for $1 \leqslant k \leqslant K$ :

1. If $\left|\lambda_{k}\right|<|\lambda|$, then $Q_{n, k}=O\left(n^{-1}\right)$.

2. If $D_{k} \leqslant D-1$, then $Q_{n, k}=O\left(n^{-1}\right)$.

3. If $D_{k}=D$ and $\left|\lambda_{k}\right|=|\lambda|$, then

$$
Q_{n, k}=\delta\left(\lambda_{k} / \lambda\right)^{n} \lambda_{k}^{1-D} W^{D-1}+O\left(n^{-1}\right) .
$$


LEMMA 2. Let $\lambda(M) \geqslant 1$ and $\left\|\mathrm{w}_{n}\right\| \leqslant w<\infty$ for all $n$. The following hold for $1 \leqslant k \leqslant K$ :

1. If $\left|\lambda_{k}\right|<|\lambda|$, then $\mathrm{s}_{n, k} / \cdot\left(\lambda^{n} C(n, D-\delta)\right)=O\left(n^{-1}\right)$.

2. If $D_{k} \leqslant D-1$, then $s_{n, k} /\left(\lambda^{n} C(n, D-\delta)\right)=O\left(n^{-1}\right)$.

3. If $\left|\lambda_{k}\right|=|\lambda|$ and $D_{k}=D$, then

$$
\begin{gathered}
\mathbf{s}_{n, k} /\left(\lambda^{n} C(n, D-\delta)\right)=\left(\lambda_{k} / \lambda\right)^{n} \lambda_{k}^{1-D} W^{D-1} \mathbf{f}_{k}\left(\lambda_{k}^{-1}\right)+O\left(n^{-1}\right), \\
\lim _{n \rightarrow \infty}\left\|\mathbf{s}_{n, k} /\left(\lambda^{n} C(n, D-\delta)\right)\right\|=\left|\lambda_{k}\right|^{1-D}\left\|W^{D-1} \mathbf{f}_{k}\left(\lambda_{k}^{-1}\right)\right\| .
\end{gathered}
$$

Furthermore, $\mathbf{f}_{k}(z)$ is analytic for $|z| \leqslant\left|\lambda^{-1}\right|$.

Remark. In part 3 of Lemmas 1 and 2 notice that $\lim _{n \rightarrow \infty}\left(\lambda_{k} / \lambda\right)^{n}$ exists if and only if $\lambda_{k}=\lambda$. Hence the following limit results hold if and only if $\lambda_{k}=\lambda$ :

$$
\lim _{n \rightarrow \infty} Q_{n, k}=\delta \lambda_{k}^{1-D} W^{D-1}, \quad \lim _{n \rightarrow \infty} s_{n, k} /\left(\lambda^{n} C(n, D-\delta)\right)=\lambda_{k}^{1-D} W^{D-1} \mathbf{f}_{k}\left(\lambda_{k}^{-1}\right) .
$$

This point is the important key to hypothesis 3 which distinguishes between the root and quotient order results of Theorems 1 and 2, because in order for the quotient order result to hold, the limits above must hold. See also Eq. (17). $\square$

We now prove Theorem 1. Start from (1) in Jordan form

$$
\mathrm{y}_{n+1}=J \mathrm{y}_{n}+\mathrm{w}_{n+1} \text {. }
$$

Let $\mathrm{y}_{n, k}$ and $\mathrm{w}_{n, k}$ be the portions of the vectors $\mathrm{y}_{n}$ and $\mathrm{w}_{n}$ corresponding to the Jordan block $J_{k}$. It may be easily verified from Eqs. (10) and (11) that

$$
\mathrm{y}_{n, k}=J_{k}^{n} \mathrm{y}_{0, k}+\mathrm{s}_{n, k},
$$

where $s_{n, k}$ was given by (10). Let

$$
\mathbf{v}_{n, k}=\mathbf{y}_{n, k} /\left(\lambda^{n} C(n, D-\delta)\right) .
$$

Substitute (12) and (9) into (13). Then

$$
\mathbf{v}_{n, k}=Q_{n, k} \mathbf{y}_{0, k}+\mathbf{s}_{n, k} /\left(\lambda^{n} C(n, D-\delta)\right) .
$$

Since $\left\|\mathbf{w}_{n}^{\prime}\right\|$ is bounded by hypothesis and $U$ is a nonsingular matrix, $\left\|\mathbf{w}_{n}\right\|$ is also bounded. Lemmas 1 and 2 may be applied. Parts 1 and 2 of the lemmas clearly show that we need to consider only those components for which $\left|\lambda_{k}\right|=|\lambda|$ and $D_{k}=D$ both hold, for otherwise $\mathbf{v}_{n, k} \rightarrow \mathbf{0}$. Apply part 3 of Lemmas 1 and 2 to those components to obtain

$$
\mathbf{v}_{n, k}=\left(\lambda_{k} / \lambda\right)^{n} \lambda_{k}^{1-D} W_{k}^{D-1}\left\{\delta \mathbf{y}_{0, k}+\mathbf{f}_{k}\left(\lambda_{k}^{-1}\right)\right\}+O\left(n^{-1}\right) .
$$

We are now ready to define the subspace hypothesis which is hypothesis 2 in Theorems 1, 2, and 3:

(16) $W_{k}^{D-1}\left\{\delta \mathbf{y}_{0, k}+\mathbf{f}_{k}\left(\lambda_{k}^{-1}\right)\right\} \neq 0$ for some $k$ such that $\left|\lambda_{k}\right|=|\lambda|$ and $D_{k}=D$.

(Recall that $\mathbf{y}_{0, k}$ and $\mathbf{f}_{k}$ in (16) come from the Jordan transform of (1). Recall also that, in Theorem 2, $\mathbf{f}_{k}=\mathbf{0}$ and $\delta=1$, always.) Note (16) implies that the full vector 
$\mathbf{v}_{n}$ has at least one component which, as $n \rightarrow \infty$ is bounded away from zero (recall that $\left.\left|\lambda_{k}\right|=|\lambda| \neq 0\right)$. By (13), the same is true for the vector $y_{n}$ and thus also for $\mathbf{y}_{n}^{\prime}$.

Since $\delta=0$ when $\lambda(M)=1$, the subspace hypothesis becomes

$$
W_{k}^{D-1} \mathbf{f}_{k}\left(\lambda_{k}^{-1}\right) \neq 0
$$

for some $k$ such that $\left|\lambda_{k}\right|=1$ and $D_{k}=D$. There are many nontrivial situations that yield $\mathrm{f}_{k}\left(\lambda^{-1}\right)=0$. In such cases Theorem 1 cannot be applied although $R\left(\mathrm{y}_{n}\right)=1$ and $Q\left(\mathrm{y}_{n}\right)=1$ may still hold. We shall not pursue this in the present paper.

To establish the root order result, take norms in (15).

$$
\left\|\mathbf{v}_{n, k}\right\| \leqslant|\lambda|^{1-D}\|W\|^{D-1}\left\{\delta\left\|\mathbf{y}_{0, k}\right\|+\left\|\mathrm{f}_{k}\left(\lambda_{k}^{-1}\right)\right\|\right\}+O\left(n^{-1}\right) .
$$

Thus $\mathbf{v}_{n}$ is bounded in norm. Since $C(n, D-\delta) / n^{D-\delta} \rightarrow 1 /(D-\delta) !$ as $n \rightarrow \infty$, then

$$
\mathrm{y}_{n}^{\prime}=U^{-1} \mathbf{y}_{n}=\lambda^{n} n^{D-\delta} \mathbf{v}_{n}^{\prime}
$$

for some vector $\mathbf{v}_{n}^{\prime}$ which is bounded in norm and which by application of the subspace hypotheses is bounded away from zero for $n$ sufficiently large. Therefore,

$$
\left\|\mathbf{y}_{n}^{\prime}\right\|^{1 / n}=|\lambda| n^{(D-\delta) / n}\left\|\mathbf{v}_{n}^{\prime}\right\|^{1 / n} \rightarrow|\lambda| \quad \text { as } n \rightarrow \infty .
$$

Hence $R\left(\mathrm{y}_{n}^{\prime}\right)=\lambda(M)$.

To establish the quotient order result, we need to consider only $k=1$ (by the hypotheses of Theorem 1 and by Lemmas 1 and 2). Since $\lambda_{1}=\lambda$, then Eq. (15) becomes (see the Remark following Lemma 2)

$$
\mathbf{v}_{n, 1}=\lambda^{1-D} W^{i-1}\left\{\delta \mathbf{y}_{0,1}+\mathrm{f}_{1}\left(\lambda^{-1}\right)\right\}+O\left(n^{-1}\right) .
$$

An application of the subspace hypothesis shows that $\mathbf{v}_{n, 1}$ has a nonzero limit as $n \rightarrow \infty$. Thus there is some vector $\mathbf{v}_{n}^{\prime}$, with a nonzero limit, for which

$$
\begin{gathered}
\mathbf{y}_{n}^{\prime}=\lambda^{n} n^{D-\delta} \mathbf{v}_{n}^{\prime}, \\
\frac{\left\|\mathbf{y}_{n+1}^{\prime}\right\|}{\left\|\mathbf{y}_{n}^{\prime}\right\|}=|\lambda|\left(\frac{n+1}{n}\right)^{D-\delta} \frac{\left\|\mathbf{v}_{n+1}^{\prime}\right\|}{\left\|\mathbf{v}_{n}^{\prime}\right\|} \rightarrow|\lambda| \quad \text { as } n \rightarrow \infty
\end{gathered}
$$

Hence $Q\left(\mathrm{y}_{n}^{\prime}\right)=\lambda(M)$ which completes the proof of Theorem 1.

The proof of Theorem 2 is a minor modification of the proof of Theorem 1, with $s_{n, k}=0$, and is omitted.

5. Proofs of the Lemmas. The statements of Lemmas 1 and 2 were given in Section 4 and will not be repreated here. Fix $k$ for $1 \leqslant k \leqslant K$. Let $\lambda_{k} \neq 0$, since the results are trivial otherwise. Recall that $\delta=0$ if $|\lambda|=1$ and $\delta=1$ otherwise. Let $n \geqslant D_{k}$. Then

$$
J_{k}^{n}=\left(\lambda_{k} I+W\right)^{n}=\sum_{m=0}^{D_{k}-1} \lambda_{k}^{n-m} C(n, m) W^{m},
$$




$$
J_{k}^{n}=\lambda_{k}^{n} C(n, D-\delta) \sum_{m=0}^{D_{k}-1} \lambda_{k}^{-m} \frac{C(n, m)}{C(n, D-\delta)} W^{m}
$$

Proof of Lemma 1. Substituting (18) into (9) yields

$$
Q_{n, k}=\left(\frac{\lambda_{k}}{\lambda}\right)^{n} \quad \sum_{m=0}^{D_{k}^{-1}} \lambda_{k}^{-m} \frac{C(n, m)}{C(n, D-\delta)} W^{m}
$$

Since

$$
C(n, m) / C(n, D-\delta)=O\left(n^{m-D+\delta}\right) \leqslant O\left(n^{D_{k}-D}\right) \quad \text { for } 0 \leqslant m \leqslant D_{k}-1,
$$

then

$$
\left\|Q_{n, k}\right\| \leqslant\left|\lambda_{k} / \lambda\right|^{n} O\left(n^{D_{k}-D}\right)
$$

1. If $\left|\lambda_{k}\right|<|\lambda|$, then $\left|\lambda_{k} / \lambda\right|^{n} O\left(n^{D_{k}-D}\right)=O\left(n^{-1}\right)$. Thus $Q_{n, k}=O\left(n^{-1}\right)$, as desired.

2. If $D_{k} \leqslant D-1$, then $O\left(n^{D_{k}-D}\right) \leqslant O\left(n^{-1}\right)$ while $\left|\lambda_{k} / \lambda\right|^{n} \leqslant 1$. Thus $Q_{n, k}=$ $O\left(n^{-1}\right)$, as desired.

3. If $D_{k}=D$ and $\left|\lambda_{k}\right|=|\lambda|$, then

$$
\left(\frac{\lambda_{k}}{\lambda}\right)^{n} \sum_{m=0}^{D_{k}-2} \lambda_{k}^{-m} \frac{C(n, m)}{C(n, D-\delta)} W^{m}=O\left(n^{-1}\right)
$$

by the proof of part 2 above. Hence $Q_{n, k}$ is dominated by the term $m=D_{k}-1$; that is,

$$
Q_{n, k}=\left(\frac{\lambda_{k}}{\lambda}\right)^{n}\left\{\lambda_{k}^{1-D} \frac{C(n, D-1)}{C(n, D-\delta)} W^{D-1}\right\}+O\left(n^{-1}\right) .
$$

Since $\delta=0$ or $\delta=1$, then

$$
C(n, D-1) / C(n, D-\delta)=\delta+O\left(n^{-1}\right) .
$$

Therefore

$$
Q_{n, k}=\delta\left(\lambda_{k} / \lambda\right)^{n} \lambda_{k}^{1-D} W^{D-1}+O\left(n^{-1}\right),
$$

which completes the proof of Lemma 1. $\square$

Proof of Lemma 2. Write

$$
\mathrm{t}_{n, k}=\sum_{i=D_{k}}^{n} J_{k}^{i} \mathbf{w}_{n-i, k} .
$$

Recall (10) and apply $\left\|\mathbf{w}_{n}\right\| \leqslant w$. Hence

$$
\left\|\mathrm{s}_{n, k}-\mathrm{t}_{n, k}\right\| \leqslant w \sum_{i=0}^{D_{k}-1}\left\|J_{k}\right\|^{i}=O(1)
$$

Hence

$$
\lim _{n \rightarrow \infty}\left\|\mathbf{s}_{n, k}-\mathbf{t}_{n, k}\right\| /\left(\lambda^{n} C(n, D-\delta)\right)=0
$$


Thus, it suffices to consider $\mathrm{t}_{n, k}$ instead of $\mathrm{s}_{n, k}$. Denote

$$
\mathbf{q}_{n, k}=\mathbf{t}_{n, k} /\left(\lambda^{n} C(n, D-\delta)\right) \text {. }
$$

Substitute (18) with $n=i$ and (19) into (20). Interchange the order of summation to obtain

$$
\mathrm{q}_{n, k}=\sum_{m=0}^{D_{k}^{-1}} W^{m}\left\{\sum_{i=D_{k}}^{n} \lambda_{k}^{i-m} \lambda^{-n} \frac{C(i, m)}{C(n, D-\delta)} \mathrm{w}_{n-i, k}\right\}
$$

1. Suppose that $\left|\lambda_{k}\right|<|\lambda|$. Consider $\tau_{k} \geqslant 1$ such that $\left|\lambda_{k}\right|<\left|\tau_{k}\right| \leqslant|\lambda| . \quad\left(\tau_{k}\right.$ will be picked later.) Let $b_{n, k}$ denote the vector

$$
\mathrm{b}_{n, k}=\sum_{m=0}^{D_{k}-1} \lambda_{k}^{-m} W^{m} \sum_{i=D_{k}}^{n} C(i, m)\left(\frac{\lambda_{k}}{\tau_{k}}\right)^{i} \tau_{k}^{i-n} \mathrm{w}_{n-i, k} .
$$

Then Eq. (21) may be written as

$$
\mathrm{q}_{n, k}=\left(\frac{\tau_{k}}{\lambda}\right)^{n} \mathrm{~b}_{n, k} / C(n, D-\delta) .
$$

Consider the functions $\psi_{n}(z)=\sum_{i=0}^{n} z^{i}$ and $\psi(z)=\sum_{i=0}^{\infty} z^{i}$. Since $\lim _{n \rightarrow \infty} \psi_{n}(z) \equiv$ $\psi(z)$ is analytic for $|z|<1$, then $\lim _{n \rightarrow \infty} \psi_{n}^{(m)}(z) \equiv \psi^{(m)}(z)$ is also analytic, and thus also absolutely convergent for $|z|<1$ (superscript denotes differentiation). Thus

$$
\left|\psi_{n}^{(m)}(z)\right| \leqslant \psi^{(m)}(|z|) \text { for }|z|<1 .
$$

It is not hard to verify that

$$
\sum_{i=m}^{n} C(i, m) z^{i}=\frac{1}{m !} z^{m} \psi_{n}^{(m)}(z) .
$$

Since $0 \leqslant m \leqslant D_{k}-1,\left|\lambda_{k} / \tau_{k}\right|<1,\left|\tau_{k}\right|^{i-n} \leqslant 1$ for $D_{k} \leqslant i \leqslant n$, and $\left\|\mathbf{w}_{n-i, k}\right\| \leqslant w$, then the norm of (22) yields

$$
\left\|\mathbf{b}_{n, k}\right\| \leqslant w \sum_{m=0}^{D_{k}-1}\left|\lambda_{k}\right|^{-m}\left\|W^{m}\right\| \frac{1}{m !} \psi^{(m)}\left(\left|\lambda_{k}\right| /\left|\tau_{k}\right|\right) \equiv b .
$$

Clearly, $b=O(1)$. Take norms in (23) to obtain

$$
\left\|\mathbf{q}_{n, k}\right\| \leqslant b\left|\frac{\tau_{k}}{\lambda}\right|^{n} / C(n, D-\delta) .
$$

If $|\lambda|>1$, choose $\tau_{k}<|\lambda|$; in this case $\mathrm{q}_{n, k}=O\left(n^{-1}\right)$. If $|\lambda|=1$, then $\delta=0$; in this case $1 / C(n, D-\delta)=O\left(n^{-1}\right)$ and thus $\mathrm{q}_{n, k}=O\left(n^{-1}\right)$. This establishes part 1 of Lemma 2.

2. Suppose that $1 \leqslant D_{k} \leqslant D-1$. In view of part 1 , it is only necessary to prove part 2 when $\left|\lambda_{k}\right|=|\lambda|$. Note that $0 \leqslant m \leqslant D_{k}-1 \leqslant D-2$. If $|\lambda|=1$, then

$$
\begin{aligned}
\sum_{i=D_{k}}^{n} \frac{C(i, m)}{C(n, D-\delta)}|\lambda|^{i-n} & \leqslant \sum_{i=m}^{n} \frac{C(i, m)}{C(n, D)}=\frac{C(n+1, m+1)}{C(n, D)} \\
& \leqslant O\left(n^{m+1-D}\right) \leqslant O\left(n^{-1}\right) .
\end{aligned}
$$

If $|\lambda|>1$, then 


$$
\sum_{i=D_{k}}^{n} \frac{C(i, m)}{C(n, D-\delta)}|\lambda|^{i-n} \leqslant \frac{C(n, D-2)}{C(n, D-1)} \cdot\left(1+|\lambda|^{-1}+|\lambda|^{-2}+\cdots\right) \leqslant O\left(n^{-1}\right) .
$$

Thus, for $|\lambda| \geqslant 1$ and for $0 \leqslant m \leqslant D-2$,

$$
\sum_{i=D_{k}}^{n} \frac{C(i, m)}{C(n, D-\delta)}|\lambda|^{i-n}=O\left(n^{-1}\right) .
$$

Take norms in (21), apply $\left|\lambda_{k}\right|=|\lambda|,\left\|\mathbf{w}_{n-i, k}\right\| \leqslant w$, and (26) to obtain $\left\|\mathbf{q}_{n, k}\right\| \leqslant$ $O\left(n^{-1}\right)$. This establishes part 2 of Lemma 2 .

3. Suppose that $\left|\lambda_{k}\right|=|\lambda|$ and $D_{k}=D$. Then (21) becomes

$$
\begin{aligned}
\mathbf{q}_{n, k} & =\sum_{m=0}^{D-1} \lambda^{-n} \lambda_{k}^{n-m} W^{m} \sum_{i=D}^{n} \lambda_{k}^{i-n} \frac{C(i, m)}{C(n, D-\delta)} \mathbf{w}_{n-i, k} \\
& =\left(\frac{\lambda_{k}}{\lambda}\right)^{n} \sum_{m=0}^{D-1} \lambda_{k}^{-m} W^{m} \sum_{i=0}^{n-D} \lambda_{k}^{-i} \frac{C(n-i, m)}{C(n, D-\delta)} \mathbf{w}_{i, k} .
\end{aligned}
$$

We may write

$$
\mathrm{q}_{n, k}=\left(\frac{\lambda_{k}}{\lambda}\right)^{n} \sum_{m=0}^{D-1} \lambda_{k}^{-m} W^{m} \mathbf{f}_{k, m, n}\left(\lambda_{k}^{-1}\right)
$$

where $f_{k, m, n}(z)$ is the vector polynomial

$$
\mathbf{f}_{k, m, n}(z)=\sum_{i=0}^{n-D} z^{i} \frac{C(n-i, m)}{C(n, D-\delta)} \mathbf{w}_{i, k}
$$

Hence

$$
\left\|\mathrm{f}_{k, m, n}(z)\right\| \leqslant w \sum_{i=D}^{n}|z|^{n-i} \frac{C(i, m)}{C(n, D-\delta)} .
$$

Let $z=\lambda_{k}^{-1}$. Since $\left|\lambda_{k}\right|=|\lambda| \geqslant 1$, we may apply (26) to (29) and obtain

$$
\left\|\mathbf{f}_{k, m, n}\left(\lambda_{k}^{-1}\right)\right\|=O\left(n^{-1}\right) \text { for } 0 \leqslant m \leqslant D-2 .
$$

Thus $\mathbf{q}_{n, k}$ in (27) is dominated by the term with $m=D-1$. Evaluate (29) with $m=D-1$ for $|z| \leqslant\left|\lambda^{-1}\right|$. If $|\lambda|>1$, then $\delta=1$ and

$$
\begin{aligned}
\left\|\mathrm{f}_{k, D-1, n}(z)\right\| & \leqslant w \frac{C(n, D-1)}{C(n, D-1)} \sum_{i=0}^{n}|\lambda|^{i-n} \\
& \leqslant w\left(1+|\lambda|^{-1}+|\lambda|^{-2}+\cdots\right)=\frac{w|\lambda|}{|\lambda|-1} .
\end{aligned}
$$

On the other hand if $|\lambda|=1$, then $\delta=0$ and

$$
\begin{aligned}
\left\|\mathrm{f}_{k, D-1, n}(z)\right\| & \leqslant w\left\{\sum_{i=D-1}^{n} C(i, D-1)-1\right\} / C(n, D) \\
& =w\{C(n+1, D)-1\} / C(n, D) \leqslant w(n+1) /(n+1-D)=O(1) .
\end{aligned}
$$

Thus $\mathrm{f}_{k, D-1, n}(z)$ is bounded in norm uniformly in $n$ for $|z| \leqslant\left|\lambda^{-1}\right|$. Hence

$$
\mathbf{f}_{k}(z) \equiv \lim _{n \rightarrow \infty} \mathbf{f}_{k, D-1, n}(z)
$$


is analytic for $|z| \leqslant\left|\lambda^{-1}\right|$. (In fact $f_{k}(z)$ is analytic for $|z| \leqslant 1$ when $|\lambda|=1$ and for $|z|<1$ when $|\lambda|>1$.) Equation (27) may be written as

$$
\mathbf{q}_{n, k}=\left(\lambda_{k} / \lambda\right)^{n} \lambda_{k}^{1-D} W^{D-1} \mathbf{f}_{k}\left(\lambda_{k}^{-1}\right)+O\left(n^{-1}\right)
$$

To complete the proof, take norms, recall that $\left|\lambda_{k}\right|=|\lambda|$, and take the limit as $n \rightarrow$ $\infty$. ․

Acknowledgment. We are indebted to D. Heller, CMU, A. Hindmarsh, LLL, H. T. Kung, CMU, and H. Woźniakowski, CMU and the University of Warsaw, for major suggestions for improving this paper.

Department of Mathematics

Arizona State University

Tempe, Arizona 85281

Department of Computer Science

Carnegie-Mellon University

Pittsburgh, Pennsylvania 15213

ALAN FELDSTEIN \& R. M. FIRESTONE [67], Hermite Interpolatory Iteration Theory and Parallel Numerical Analysis, Appl. Math. Dept. Report, Brown Univ., October 1967.

ALAN FELDSTEIN \& R. M. FIRESTONE [69], "A study of Ostrowski efficiency for composite iteration algorithms," Proc. Nat. Conf. Assoc. Comp. Mach., 1969, pp. 147-155.

ALAN FELDSTEIN \& J. F. TRAUB [74], Order of Vector Recurrences with Applications to Nonlinear Iteration, Parallel Algorithms, and the Power Method, Comput. Sci. Dept. Report, Carnegie-Mellon Univ., December 1974.

J. HERZBERGER [74], "Über Matrixdarstellungen für Iterationsverfahren bei nichtlinearen Gleichungen," Computing, v. 12, 1974, pp. 215-222.

A. C. HINDMARSH [72], "Optimality in a class of root-finding algorithms," SIAM J. Numer. Anal., v. 9, 1972, pp. 205-214. MR 47 \#275.

H. T. KUNG \& J. F. TRAUB [74], "Computational complexity of one-point and multipoint iteration," Complexity of Computation, (SIAM-AMS Proceedings, vol. 7), edited by R. M. Karp, Amer. Math. Soc., Providence, R. I., 1974, pp. 149-160.

W. L. MIRANKER [69], "Parallel methods for approximating the root of a function," IBM J. Res. Develop., v. 13, 1969, pp. 297-301. MR 39 \#1109.

J. R. RICE [71], "Matrix representations of nonlinear equation iterations-Application to parallel computation,” Math. Comp., v. 25, 1971, pp. 639-647. MR 46 \# 2850.

G. S. SHEDLER [67], "Parallel numerical methods for the solution of equations," Comm. $A C M$, v. 10, 1967, pp. 286-291. MR $39 \# 2321$.

J. F. TRAUB [64], Iterative Methods for the Solution of Equations, Prentice-Hall, Englewood Cliffs, N. J., 1964. MR 29 \# 6607.

J. F. TRAUB \& H. WOŹNIAKOWSKI [76], "Strict lower and upper bounds on iterative computational complexity," Analytic Computational Complexity, edited by J. F. Traub, Academic Press, New York, 1976.

R. S. VARGA [62], Matrix Iterative Analysis, Prentice-Hall, Englewood Cliffs, N. J., 1962. MR 28 \# 1725. 